\title{
Progressive Familial Heart Block, Type la
}

National Cancer Institute

\section{Source}

National Cancer Institute. Progressive Familial Heart Block, Type la. NCI Thesaurus.

Code C126651.

An autosomal dominant inherited cardiac bundle branch disorder which can progress to complete heart block. 